\title{
EL MÉTODO BIOGRÁFICO EN \\ INVESTIGACIÓN SOCIAL: \\ POTENCIALIDADES Y LIMITACIONES \\ DE LAS FUENTES ORALES Y \\ LOS DOCUMENTOS PERSONALES
}

\author{
Alexia Sanz Hernández \\ Profesora Titular de Sociología, Universidad de Zaragoza
}

\section{RESUMEN}

La reconstrucción biográfica es un juego de intersubjetividades que emerge esencialmente de la persona y de su testimonio, ya sea oral u escrito. Memoria, identidad y sociabilidad son igualmente dispositivos desde los cuales hay que entender las potencialidades y limitaciones de los documentos orales y materiales de vida, fuentes sobre las que se fundamenta la investigación biográfica. El trabajo muestra, de un modo práctico o didáctico, cómo orientarse en este proceso.

PALABRAS CLAVE: Investigación social, técnicas cualitativas, método biográfico, historias de vida, identidad y memoria.

\section{SUMMARY}

The biographical reconstruction is a game of intersubjectivities that emerges essentially from the person and her testimony, either oral or written. Memory, identity and sociability are also devices from which there are to understand the potentialities and limitations of the oral and material documents of life, sources on which the biographical investigation is based. This paper is a didactic essay about how to make it.

KEY WORDS: Social studies, qualitative methods, biographical research, life-history, identity, memory.

La reconstrucción biográfica emerge esencialmente de una persona y de su testimonio, ya sea oral u escrito, y de su interacción con el que lo retoma, interpreta y rehace (aún cuando este sea el mismo protagonista de los hechos que asume el rol de escritor como en el caso de las autobiografías), de modo que el 
juego de intersubjetividades va a ser una dinámica inherente y permanentemente presente. Igualmente emerge un mecanismo enmarañado, complejo, dinámico, selectivo y efectivo, cual es la memoria. A lo largo de este texto pretendo incorporar algunas reflexiones de interés que no pueden obviarse en la investigación biográfica, enfatizando determinados rasgos metodológicos claramente enmarcados en la disciplina de la antropología y la sociología, y relativos a un método que se centra esencialmente en la atención a dimensiones psicológicas y contextuales del individuo por encima de todo.

\section{CUADRO 1: FUNDAMENTOS DE LA RECONSTRUCCIÓN BIOGRÁFICA}

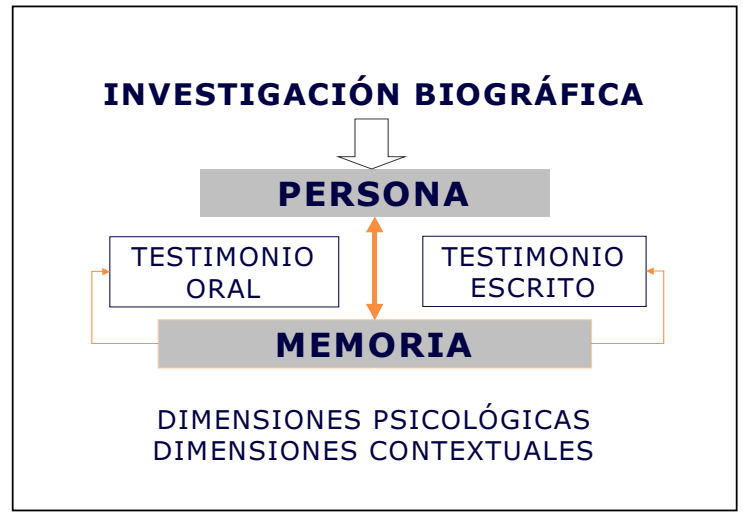

\section{LA INVESTIGACIÓN BIOGRÁFICA}

En la larga historia de las ciencias sociales, la aproximación biográfica ha sido una de las constantes que, bajo perspectivas epistemológicas e intencionalidades teóricas diversas, ha llegado a nuestros días con un vigor inusitado y desde procedencias disciplinares diversas. El interés por la descripción biográfica no es reciente ${ }^{1}$ aunque el despegue de la metodología de los relatos de vida coincide con el apogeo de la Escuela de Chicago en los años veinte. Desde la época del American Bureau of Ethnology y la ingente producción de los Indian Portraits de los etnólogos y periodistas norteamericanos hasta las

1 La obra de 1989 de DeNZIN, Interpretive Biography, recoge una interesante visión de conjunto de antecedentes y estrategias. 
piezas maestras de la Antropología, ha habido un uso continuo de las fuentes orales en la investigación biográfica ${ }^{2}$. Lo mismo ocurre en Sociología, área de conocimiento en la que existe una interesante tradición metodológica ${ }^{3}$ sobre el uso de los llamados documentos personales y las historias de vida, aunque ensombrecida por el pretendido «mito de la cientificidad sociológica» que redujo el protagonismo de la metodología cualitativa en décadas pasadas.

Pero no sólo antropólogos o sociólogos han hecho uso de esta metodología; la historia toma prestado el método de la investigación oral para crear un nuevo corpus documental. Así nace la historia oral, con carácter de ciencia auxiliar inicialmente, cuyo objetivo es construir archivos orales, lo cual respondía en un principio más a la pretensión de obtener información de los propios testigos vivos que a la de suplementar o reemplazar datos de las fuentes escritas; sin embargo, desde esta aproximación, la oralidad está cobrando un auge especial y la introducción de esta nueva técnica ha supuesto un cuestionamiento de la historiografía que tendía a dar prioridad al papel de los actores dominantes en la interpretación del pasado frente a los dominados, la gente común ${ }^{4}$.

Por su parte, la Psicología social ha empleado las biografías como historias clínicas en las que se perfila el entorno social de cada individuo analizado. El enfoque es fundamentalmente evolutivo y pretende mostrar la perspectiva del desarrollo vital en el contexto histórico, todo ello a través de la reconstrucción de las experiencias vitales.

Todas estas disciplinas caminan juntas en el uso de las mismas técnicas y fuentes que como parecen estar confirmando las investigaciones recientes, surgen como material por excelencia para quien quiera estudiar las transformaciones no sólo del individuo sino también de su grupo primario y su entorno sociocultural inmediato. Igualmente puede acercarnos al conocimiento del cambio social, los procesos históricos de las relaciones socioestructurales, las trayectorias de vida, la descripción con profundidad de las relaciones sociales, sus contradicciones o

2 La visión interna de las sociedades aprehendidas por el relato rememorativo, la entrevista, la biografía y la autobiografía ha sido lo propio de los antropólogos, tal y como lo demuestran las grandes autobiografías etnográficas de Leo Simmons, Sun chief (1942) y Oscar Lewis, Los hijos de Sánchez (1961).

3 Concluida la primera guerra mundial, la Escuela de Chicago llevó a cabo interesantes análisis de las minorías inmigrantes a través de relatos de vida, como la obra de F. Zaniecki y W.I. Thomas Polish Peasant (1918-1920). También de este periodo es la publicación de las primeras autobiografías indias como las realizadas por el antropólogo Paul Radin: The autobiography of a Winebago Indian (1920) o Crashing thunder, de 1926.

4 M. N. Bourguet, L. Valensi y N. Watchel editaron en 1990 una interesante recopilación de artículos en una obra titulada Between Memory and History. 
su movimiento histórico, y es una herramienta excelente para quien quiera con fines formativos fomentar su utilización o ilustrar dichos procesos.

Puede tratarse únicamente de una moda pasajera o perpetuarse como una auténtica corriente metodológica y analítica. Lo cierto es que, en los últimos años, disciplinas dispares caminan juntas en el objetivo de plantear y desarrollar una nueva manera de hacer teoría y ciencia social.

La técnica de elaboración de relatos de vida y de historias de vida se inserta en una metodología más amplia denominada el método biográfico, junto a cuya denominación necesariamente emergen conceptos como investigación etnográfica, estudio de casos, observación participante, etc. Este método puede aglutinar la estrategia metodológica de la conversación y narración y la revisión documental de autobiografías, biografías, narraciones personales, cartas, diarios, fotos, etc. Conjuga de este modo fuentes orales con fuentes documentales personales con el propósito doble de, primero, captar los mecanismos que subyacen a los procesos que utilizan los individuos para dar sentido y significación a sus propias vidas, y segundo, mostrar un análisis descriptivo, interpretativo, y necesariamente sistemático y crítico de documentos de «vida».

La primera consideración a realizar se relaciona con la gran cantidad de términos y usos que se vinculan con «lo biográfico». Los términos más utilizados son el de «biografía» y «autobiografía»; la diferencia entre ellos se encuentra en la existencia o no de la figura mediadora de un agente externo que construya finalmente el relato. A la anterior se añade la distinción entre life story que corresponde a la historia de una vida tal y como la persona que la ha vivido la cuenta, y la life history vinculada al estudio de caso referido a una persona dada, comprendiendo no sólo sus relatos de vida sino cualquier otro tipo de información o documentación adicional que permita la reconstrucción de dicha biografía de la forma más exhaustiva y objetiva posible» ${ }^{5}$. En castellano estaríamos hablando de relatos de vida e historias de vida respectivamente. Un termino más es el de «biograma» que se refiere a «los registros biográficos de carácter más sucinto y que suponen la recopilación de una amplia muestra de biografías personales a efectos comparativos ${ }^{6}{ }^{6}$. Pujadas propone la siguiente delimitación terminológica.

- Documentos personales.

- Autobiografías,

- Diarios personales,

5 Pujadas, J. J. (1992), p. 13.

6 Pujadas, J. J. (1992), p. 14. 
- Correspondencia, colecciones epistolares,

- Fotografías, películas, videos y otros registro iconográficos,

- Objetos personales.

- Registros personales obtenidos por encuestas.

- Historias de vida

- de relato único,

- de relatos cruzados,

- de relatos paralelos.

- Relatos de vida sometidos a otros tratamientos analíticos

- Biogramas.

Básicamente podemos encontrarnos con dos aplicaciones del método biográfico: las historias de vida como estudios de caso, o bien la técnica de los relatos biográficos múltiples. En el primer caso nos encontramos ante «un relato autobiográfico, obtenido por el investigador mediante entrevistas sucesivas en las que el objetivo es mostrar el testimonio subjetivo de una persona, en el que se recojan tanto los acontecimientos como las valoraciones que dicha persona hace de su propia existencia» ${ }^{7}$. En esta modalidad el investigador es únicamente un introductor de la obra, que retoca y matiza la presentación final del relato tras ordenar la información obtenida en las prolongadas sesiones con el informante.

Hay que reflexionar acerca de las matizaciones y dimensiones diferenciales que incorpora el hecho de proceder a la elaboración de historias de vida, por un lado, o de autobiografías, por otro. Estas segundas son producto de la propia voluntad de su autor y no existe un agente externo inductor del proceso, pero a su vez el autor se ve influenciado por una serie de sesgos o filtros heurísticos de los que hablaremos más adelante y que tamizan su aportación.

En ciencias sociales se utilizan también con frecuencia los «relatos biográficos múltiples» como si fuese una forma de encuesta en la que las decisiones muestrales pueden asentarse en criterios de representatividad junto a los de significatividad (por ejemplo, utilizando tipologías de sujetos a partir de variables preestablecidas o atendiendo al procedimiento de «saturación» propuesto por Denzin en 1970). Pujadas distinguía, como hemos observado, dos modalidades de este tipo de relatos, los «paralelos» y los «cruzados». Mientras que los relatos de vida paralelos se refieren a trayectorias de vida que han

7 Ibidem, pp. 47-48. 
transcurrido sin converger ni generar vínculos entre sí, los segundos aluden a las «historias de vida cruzadas de varias personas de un mismo entorno, bien sean familiares, vecinos de un barrio, o compañeros de una institución, para explicarnos a «varias voces» una misma historia» ${ }^{8}$. Se trataría de acumular una muestra más o menos amplia en función de los objetivos o intereses de la investigación con la que se puede trabajar pudiendo establecer comparaciones, categorizaciones de los informantes y, si es posible, planteando modelos teóricos validados por la información recabada, tras realizar análisis que pueden ir desde el tipológico, o de contenido, al estadístico.

\section{LA PRÁCTICA DE LA INVESTIGACIÓN BIOGRÁFICA}

La investigación biográfica es esencialmente una descripción fenomenológica que exige de cuatro habilidades procedimentales en el investigador: observar, escuchar, comparar y escribir, tal y como propone Sanmartín (2003) para la práctica de la investigación cualitativa. Esas cuatro habilidades, que hay que poner en juego, se concretan en una serie de etapas, que exigen a la par sistematicidad y cierta dosis de artesanía (tal y como recomendaba Wright Mills's). En este sentido, la obra de J. J. Pujadas de 1992 se conforma como referente obligado en los estudios basados en el método biográfico.

No obstante, todos estos planteamientos metodológicos no alcanzan su significado pleno si no es en relación con el objeto de estudio al que va a ser aplicado: la persona y los documentos de vida por ella generados o facilitados.

$8 \quad$ Ibidem, p. 83.

9 «Sea un buen artesano: evite un conjunto rígido de procedimientos. Pero sobre todo, trate de desarrollar y aplicar la imaginación sociológica. Eluda el fetichismo del método y la técnica. Impulse la rehabilitación de una artesanía intelectual no presuntuosa, y trate de convertirse en artesano usted mismo. Que cada hombre sea su propio metodólogo»: WRIGHT MiLls, C. (1959), La imaginación sociológica, México, F.C.E. p. 55. 
CUADRO 2: LA PRÁCTICA DE LA INVESTIGACIÓN BIOGRÁFICA

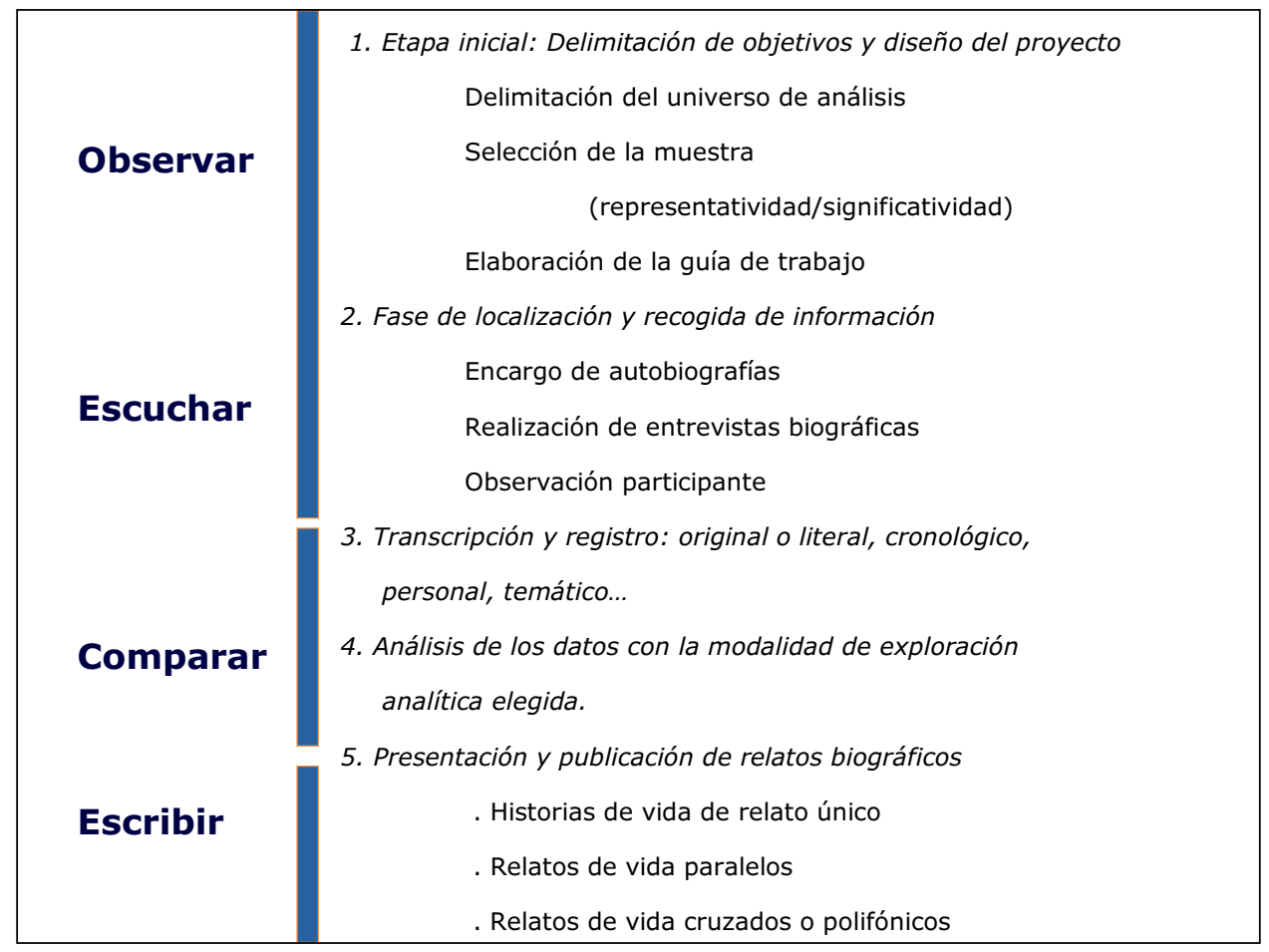

3. LA PERSONA COMO FUENTE BÁSICA DE LA INVESTIGACIÓN BIOGRÁFICA: IDENTIDAD, MEMORIA Y SOCIABILIDAD

La fuente primordial de los relatos de vida es «la persona» y el testimonio que ésta proporciona, en su doble faceta de individualidad única y sujeto histórico. En ella confluyen dimensiones psicológicas y contextuales cuya interacción genera una manera peculiar de construir y narrar su experiencia pasada, siempre en clara relación con la situación presente y los proyectos de futuro.

En la práctica, los intereses disciplinarios y la experiencia tienden a favorecer el desarrollo teórico de uno u otro de los extremos de esta cadena causal: lo individual y lo social. Sin embargo, no se puede comprender el tipo de gente que los individuos llegan a ser sin hacer referencia a las estructuras históricas donde están organizados los entornos de su vida cotidiana. 
Los vectores individuo/ colectividad interactúan estrechamente enlazados, siendo dos campos interdependientes en el documento de vida de los individuos: los cambios demográficos, técnicos, económicos y culturales modifican los sucesos vitales de los individuos y sus patrones de envejecimiento. A su vez, a través del papel que ejercen, el poder que ostentan o las ideas, sentimientos o emociones que expresan, participan en la acción histórica eligiendo $o$ influyendo en la realidad que les circunda.

Nuestra vida y nuestra personalidad dependen en gran medida de la visión que tenemos de nuestro pasado. Las experiencias históricas y los modos de existencia de los que participa el sujeto y en los que se halla inserto condicionan su comportamiento, su personalidad, la narración de su propia vida y la significación atribuida a cada experiencia vivida u oída. Los cambios en los relatos, el énfasis, los juicios de valor que surgen con la evocación de los recuerdos responden a una lógica retrospectiva que organiza los sucesos y les da un significado según la percepción global que el sujeto tiene de su vida pasada. Por eso no es infrecuente observar cómo el mismo suceso vivido por varias personas es relatado de forma muy diferente años después por los mismos testigos dependiendo de sus posteriores experiencias y destinos ${ }^{10}$.

La práctica de la investigación biográfica es impensable sin recurrir a los conceptos de «identidad» $\mathrm{y}$ «memoria», diferentes pero perfectamente imbricados.

La memoria autobiográfica se construye sobre las experiencias vividas individualmente; el sujeto es el actor y protagonista del relato y aun siendo vivencias compartidas, los eventos forman parte fundamental de su biografía individual. A esa singularidad propia del individuo se vincula la dimensión social, el espacio y el tiempo de los que relatan su historia. Las imágenes del pasado y el conocimiento recordado que les han sido transmitidos ejercen una poderosa influencia. Desde nuestra situación actual juzgamos el pasado y nos apoyamos en representaciones colectivas e interpretaciones, en principio ajenas a nosotros como personalidades individuales pero que, sin embargo, configuran la racionalización que de nuestra vida y del contexto hacemos. Además, la memoria colectiva no se nutre únicamente de la memoria construida desde las propias experiencias vividas en la trayectoria personal, sino que a

10 Yves Lequin llevó a cabo una investigación con trabajadores en Givors y realizó la siguiente observación: incluso cuando se refiere a sucesos vividos con otros y fuertemente sentidos, los relatos son extremadamente variados y siempre cruzan el prisma de la vida individual: LEQUIN Y.; METRAL J. (1980), «A la recherche d «une memoire collective: les metallugistes retraités de Givors», Annales E.S.C. p. 156. 
ésta se vincula todo el legado pasado sobre leyendas y mitos o realidades que la oralidad ha recreado, y las vivencias de contemporáneos y coetáneos.

Junto al ciclo vital individual se desarrolla otro de dimensiones más vastas: la sucesión de generaciones ${ }^{11}$. La edad estratifica a las personas y condiciona sus papeles sociales y roles a desempeñar en una comunidad, así como las relaciones interpersonales; clasifica en cierta forma a la gente en grupos o cohortes diferenciados. Pero el factor biológico no es el único que define la adscripción a una determinada generación en sentido sociológico ${ }^{12}$. Las características distintivas de una generación son, en primer lugar, la aceptación y/o parcial creación de actitudes y valores. Segundo, una participación activa o pasiva en los sucesos que les suceden, ocasionan o intentar regular, y por último, un fondo común de aspiraciones y tareas que desempeñar y cumplir.

En la práctica, la identificación de generaciones que puede realizarse no viene determinada únicamente por factores biológicos o históricos, puesto que los sucesos históricos de todos los miembros no han sido idénticos ${ }^{13}$. Las diferencias en sus historias vitales les predisponen a elaborar sus experiencias de diferentes maneras. Un ejemplo que corrobora esta afirmación es el hecho de que algunos desarrollos de carácter general fueron experimentados de diferentes maneras según los grupos de edad en distintos momentos de sus ciclos vitales y por tanto desde diferentes perspectivas. Pese a esto, el propio informante a través del uso del «nosotros» o el «esos», establece la distancia o proximidad entre los diferentes grupos de edades; los informantes se legitiman entre sí reconociéndose mutuamente como miembros o no del mismo grupo generacional.

Efectivamente, el pasado nunca se cuenta como algo no compartido; a través de los relatos se revela un tipo de sociabilidad informal, y es en esta interacción dinámica en la que se traza una memoria colectiva. La forma de recordar el pasa-

11 Lison Tolosana, C. (1983), Belmonte de los caballeros. Antropology and history in an Aragonese Communit, Princenton, New Jersey, Princenton U. P.

12 Ortega y Gasset y J. Marías proponen una definición recogida por Carmelo Lisón en la que se conceptualiza generación sociológica de la siguiente forma: «An age-group of men and women who share a common mode of existence or concept of life, who assess the significance of what happens to them at a given moment in terms of a common fund of conventions and aspirations» (LisON TOLOSANA, C. op. cit, p. 23)

13 Cada generación se encuentra con un acontecimiento en un momento diferente de su trayectoria vital. Estas divergencias suponen variaciones en el significado del suceso, en el potencial de adaptación y en las opciones y, por lo tanto, en los nexos entre la trayectoria vital. De ahí que los estudios de las variaciones históricas dentro de una única generación planteen preguntas relativas a la generalidad de sus hallazgos. 
do supone, por lo tanto, una visión sugestiva de cómo esa generación ha abordado el paso del tiempo y los cambios en él contenidos. Parafraseando al tan citado M. Halbwachs podemos decir que «la singularidad, la irreductible originalidad de los recuerdos personales se producen de hecho por el entrecruzamiento de varias series de memorias que se corresponden a los grupos variados a los que hemos pertenecido». Y así, además de la memoria autobiográfica podríamos hablar de las relaciones generacionales, la memoria de clase y la memoria nacional, cuestiones estas últimas que no voy a comentar; todos estos niveles configuran el juicio que sobre el pasado tiene el informante.

\section{PotenCIALIDADES DE LAS FUENTES ORALES Y LOS DOCUMENTOS PERSONALES}

Hasta hace unas décadas, la oralidad era la fuente de expresión por excelencia de muchas comunidades. A principios de siglo empezaron a utilizarse en EE.UU. de América las llamadas «historias de vida» ante dos circunstancias específicas: primero, la emigración masiva procedente de Europa y los problemas de adaptación que ello supuso y, en segundo lugar, la presión sobre la población india, ya en declive, del rápido proceso de modernización del país. Estos primeros trabajos estaban guiados más que nada por una preocupación de salvaguarda cultural, «recoger mientras fuera tiempo todo lo que subsistiera del pasado». Esta preocupación dió paso después a la inquietud metodológica, influidos los autores por el desarrollo y auge del método cuantitativo y el llamado "mito de la cientificidad sociológica», que D. Bertaux denuncia proponiendo una sociología de «rostro humano» que rechace la consideración de los «hechos sociales como cosas» ${ }^{14}$. Esta va a ser precisamente una de las virtualidades de la investigación biográfica: la posibilidad de contrastar entre la perspectiva macro y la micro, compensando de este modo la alta dosis de objetivismo ficticio del que hacen gala ciertas investigaciones.

Se requiere un enfoque idiográfico (es decir centrado en el individuo) y cualitativo, que implica entre otras cosas la característica de ser inductivo. Los sujetos o grupos no se reducen a variables sino que son considerados como un todo dentro de su contexto ecológico, social e histórico. Cada individuo es singular; las historias de los individuos no deben ni quedarse en el simple dato estadístico «objetivo» pero vacío ni, en caso especial, desvinculados de las condiciones contextuales de cualquier trayectoria personal. Tanto la supresión como el énfasis de esa singularidad acaban por distorsionar la

14 Véase: Marinas, J. M.; SANTAMARia, C. (1993) 
vivencia del individuo y por desmarcarlo de su marco de referencia social. Esta metodología es claramente asumida por las posiciones fenomenológicas, en tanto en cuanto se presta atención al significado social que los sujetos atribuyen al mundo que les rodea, tal y como se ha propuesto siguiendo la perspectiva interaccionista en Psicología Social: «si los hombres definen las situaciones como reales, éstas son reales en sus consecuencias».

Otra de las potencialidades de la oralidad y los documentos personales es la riqueza y profundidad de la información que puede obtenerse, en muchos casos imprevista (de acuerdo con J. Ibañez se trataría más de un proceso de descubrimiento que de búsqueda), que incorpora el carácter retrospectivo, longitudinal y subjetivo, como hemos enfatizado con anterioridad. Obviamente, la gran cantidad de datos debe ser manejada persiguiendo más un criterio de significatividad que de representatividad.

Cuando trabajamos en estudios cualitativos ni el número ni el tipo de informantes se especifica de antemano. Si nuestra finalidad con la investigación es la construcción de teorías a partir de los datos cualitativos empleando el método de la inducción analítica, deberemos recoger el mayor numero posible de casos. Incluso se ha propuesto desde la psicología elaborar muestras representativas de grupos determinados de sujetos, suponiendo que es posible obtener las historias de vida de cierto número de sus componentes; la propuesta es el estudio de los datos desde un enfoque también nomotético y no únicamente idiográfico y proceder a realizar las generalizaciones pertinentes, pero quizás esto es más criticable desde la investigación cualitativa.

Si nuestra finalidad es el conocimiento de un fenómeno en sí y por sí, la estrategia del muestreo teórico es el procedimiento más conveniente. En esta situación el número de casos carece de importancia, lo capital es el potencial de cada uno de ellos; es la propia investigación la que le dice al investigador en qué momento la información comienza a ser repetitiva o el fenómeno que se pretendía comprender está claro. Así, por ejemplo y en relación con las reconstrucciones de experiencias vividas en la trayectoria personal, descubrimos algunos hechos considerados como muy relevantes no sólo porque aparecen en muchos de los relatos, sino por la enorme emotividad que generan.

Sin embargo, al considerar la cuestión del muestreo cabe reflexionar sobre el principal inconveniente que se deriva del tratamiento de la información obtenida a través de la oralidad y los documentos de vida: la complejidad en el análisis de los datos. La técnica comprende tanto observaciones de acontecimientos como interpretaciones, juicios de valores y, en general, todo tipo de datos etnográficos, psicológicos, biológicos y culturales.

Todas estas fuentes orales junto con otras documentales como cartas, informes contemporáneos, o descripciones de periódicos, fotos y todos aquellos 
recursos que manifiestan y acompañan al testimonio del informante, así como los datos que de ellas se obtienen, no solo amplían y muestran la validez de la información obtenida, sino que gracias a ello se recoge un corpus abundante y rico en informaciones (que hace posible la triangulación informativa y metodológica). Esto constituye una de las mayores ventajas de esta técnica, pese a que en la posterior elaboración de la información, en la síntesis de la misma y su análisis, resida su mayor dificultad. Autores como Poirier, Clapier-Valladon y Raybaut proponen un análisis de contenido cuya finalidad sea hacer evidente las constantes que se dan en los relatos. El marco de análisis es abierto, es decir, no hay nada fijado a priori sino que se trata de elaborar categorías descriptivas que definen y estructuran temáticamente el relato. La apertura debe ser, por lo tanto, una constante metodológica en estas investigaciones que deben tener mucho de artesanía, en tanto en cuanto requieren de un método que dote al proceso de investigación de flexibilidad. La creatividad y la innovación dentro de la investigación conducida con procedimientos rigurosos (aunque no necesariamente estandarizados) no hacen sino enriquecer las conclusiones del estudio. Quizás, tal y como dice Deutscher ${ }^{15}$, en investigación social nos hemos preocupado más por la coherencia que por la exactitud o no de los datos; hemos aprendido «una enormidad sobre la manera de seguir un curso incorrecto con un máximo de precisión». La flexibilidad nos permite transcurrir por el serpenteo del proceso y adaptarnos a las nuevas condiciones que se van imponiendo.

El deseo de narrar ha de hacerse resurgir; ha de ser espontáneo. Por su parte, al menos en las primeras etapas de la investigación, el investigador a menudo instiga a los informantes; lo deseable es que la espontaneidad y la voluntad e interés por contar surjan naturalmente, ya que a través de las historias de vida, o las autobiografías, las personas que por su lugar en la sociedad nunca habrían podido expresarse pueden tomar la palabra, produciéndose de esta manera un cambio cualitativo relevante en las características socioculturales de los sujetos que narran su vida. El historiador Ronald Fraser define la «historia teleológica» como «la tendencia a enfocar la investigación sólo en términos de lo que logró imponerse»». Al dar oportunidad a los individuos que vivieron y participaron en los acontecimientos de su vida cotidiana, o en los eventos históricos, de relatar sus propia trayectoria vital, podríamos incluso restablecer las contradicciones y ambigüedades de muchas situaciones sociohistóricas ${ }^{16}$.

15 Deutscher, I. (1969), «Looking backward: case studies on the progress of methodology in sociological research», The American Sociologist, 4, pp. 34-42.

16 Fraser, R. (1993), «La Historia Oral como historia desde abajo», Ayer, $n^{\circ} 12$, Madrid, Marcial Pons, pp. 79-92. 
Las narraciones se confrontan en numerosas ocasiones con las historias objetivas de las instituciones y esa contrastación puede llevarnos a determinar hasta qué punto la información que nos plantean unas determinadas fuentes y metodologías es similar, en qué aspectos se complementan, o bien, y cuando esa contrastación no se soporta, intentar comprender las incongruencias, paradojas o nueva información que surja.

Fue precisamente la cuestión de la memoria la que abrió el debate en torno a las dudas acerca de la credibilidad de la documentación oral; el interés de los investigadores más positivistas se centró en descubrir las condiciones que la memoria podía y debía cumplir para satisfacer sus demandas y requerimientos. El trabajo de 1978 de Paul Thompson, The voice of the past, trata de demostrar que la memoria no es una fuente tan pobre ni frágil como generalmente se ha creído; su argumento se basaba en la experiencia e intento en Psicología Social de definir la naturaleza biológica de la memoria y sus mecanismos funcionales. Análisis de laboratorio confirmaron que de hecho es como si después de un periodo relativamente breve de tiempo (sólo unos pocos días), durante los cuales el individuo retiene una imagen muy detallada del suceso vivido, el recuerdo entra en un proceso de selección y organización antes de ser estampado ya indeleblemente en la memoria ${ }^{17}$.

\section{LIMITACIONES METODOLÓGICAS DE LA ORALIDAD Y LOS DOCUMENTOS PER- SONALES}

Las limitaciones metodológicas en la investigación surgen de la propia naturaleza de la oralidad y los documentos materiales de vida, de la interacción investigador-informante, y de la esencia de la memoria. Veamos algunas de ellas.

En el caso de utilizar la entrevista como estrategia metodológica, el procedimiento de la reconstrucción de la historia oficial tal como es presentada por los documentos y siguiendo el tiempo histórico se entiende que es prioritario, puesto que esto permite recurrir a los puntos de referencia abstractos que dicho discurso aporta, dotando al investigador de un primer soporte común para el diálogo. El tiempo histórico puede ser una buena guía. Autores como Nor-

17 Uno de los experimentos más significativos fue llevado a cabo con jóvenes madres. Se mostraba cómo el relato acerca del nacimiento y los primeros días del recién nacido variaban en los primeros meses siguientes al nacimiento pero después se estabilizaba; los recuerdos de los gestos y las prácticas y los pequeños detalles se recordaban exactamente, y todas las narraciones repetidas eran exactas y marcadamente precisas. 
man K. Denzin argumentan a favor de esta inicial construcción de una historia objetiva en primer lugar para así, después, entrecruzar con la subjetiva y encuadrar ésta con los datos y acontecimientos específicos; sin embargo, esto presenta en muchos casos una dificultad enorme: el concepto de tiempo que subyace en los relatos rehuye la historia en muchas ocasiones. El análisis que llevan a cabo los historiadores presenta una imagen lineal con puntos de inflexión, crisis, pero siempre con una secuencia de progresión y avance unidireccional que va del pasado al presente. No es ésta la dimensión temporal que subyace en los relatos que constituyen el fundamento de la oralidad. La memoria autobiográfica y la colectiva no es lineal sino temática y simbólica; las idas y venidas son continuas. El narrador se esfuerza en las primeras etapas, por seguir una sucesión lógica e histórica (a veces guiado por el entrevistador excesivamente influido también por su formación histórica), por imponer a su oralidad una disciplina y orden; esta intención queda olvidada cuando se avanza en el estudio y aumenta la profundidad del relato, al mismo tiempo que se crean vínculos entre el entrevistador y el narrador.

El tiempo individual y comunitario no sigue un eje cronológico con intervalos claramente marcados sino que se dilata o contrae. Así por ejemplo, en ocasiones las referencias explícitas a situaciones o periodos duros son más bien escasas y breves. Se diría que el individuo o la comunidad quiere olvidar; sin embargo, es selectiva en ese olvido: los relatos un tanto épicos son frecuentes y se repiten continuamente encerrando en sí el orgullo de los narradores por haber sobrevivido ${ }^{18}$. Por ello puede resultar más sencillo partir del presente del orador puesto que la situación actual es resultado de la trayectoria pasada.

El investigador puede seguir dos líneas de actuación en la recogida de los datos; o bien, establecer una dualidad en la que se supone que el narrador no se habría explicado sin el estímulo que supone las preguntas del investigador, o la creación, en segundo lugar, de un espacio que favorezca el conocimiento profundo del Otro, y donde sea posible realizar transcripciones neutras y «objetivas» sin intervenir ni instigar al informante en sus relatos. Es en esta segunda situación en la que nos es posible dilucidar la naturaleza de la dimensión temporal que encierran los relatos.

Hay diferentes posturas ante el valor del material que proporcionan las fuentes orales y los documentos personales; desde los que opinan que no merece suficiente confianza, hasta los que creen que es una fuente de introspección que muy pocos métodos de investigación pueden igualar. Tanto unos como otros no deben obviar algunas cuestiones como las siguientes.

18 SANZ, M. A. (2000) 
La «exactitud de la información»a través de los recuerdos biográficos puede estar potencialmente viciada por la pérdida de memoria, los recuerdos selectivos y la deseabilidad social. Pueden silenciarse acontecimientos o periodos que representan regresión o fracaso social, generar relatos con raíces imaginarias para cubrir lagunas existentes o adornar la narración alimentando así el ego del narrador.

En otras ocasiones existe un «confuso perfil cronológico» de los sucesos punteado por una exactitud esporádica del detalle. Destaca también la exactitud de la información concerniente a las vivencias tempranas que contrasta con el desdibujamiento de la memoria de los últimos años. Igualmente podemos encontrar anacronismos, mezclas y fusión de hechos o acontecimientos y personajes. Puede darse el caso de que los relatores sistemáticamente combinen elementos de dos sucesos, siendo una confusión no atribuible necesariamente a lapsos de memoria de personas mayores dado que a veces ocurre en los relatos de otros informantes. Otras veces no se limita a una confusión y mezcla de elementos, sino la creación de un único evento que se ve relativamente independiente de su contexto histórico real. A menudo este sincretismo está en conexión con sucesos cuyas descripciones se refieren a tiempos tempranos que los hablantes no vivieron personalmente.

Callan, comienzan algún suceso y luego lo silencian, cambian el sujeto, regresan al presente. Llega a ser difícil para el entrevistador seguir el cursos de sus vidas, situar detalles en orden cronológico. En ocasiones el edificio autobiográfico comienza a desmoronarse y el investigador insiste en las preguntas con gran esfuerzo mental para poder formar el rompecabezas y ordenar los fragmentos fechados sólo a pinceladas. Se rompe el hilo cronológico.

El protagonista, (ya sea narrador, ya escritor o generador de cualquier otro documento) tiene sus propios objetivos y posiblemente filtra la información que ofrece utilizando algunos de los más conocidos filtros heurísticos o sesgos. En el intercambio verbal se suceden exageraciones, atribuciones causales e interpretaciones de los hechos que esconden la necesidad de autojustificación y legitimación de las propias acciones, de autocensura o vanidad; se hace uso del pasado con diferentes propósitos. Todo ello puede llevar al investigador a descubrir un tipo ideal y recreado más que un tipo real. A ello se une el hecho de que las personas dicen y hacen cosas diferentes en distintas situaciones.

La percepción del sujeto sobre su propia situación en la jerarquización social y su autoconcepto intervienen en el posicionamiento que el individuo va a asumir a la hora de participar en una investigación biográfica. Cuando se trabaja con informantes de clases sociales o género colocados inferiormente en la estratificación social, podemos observar una falta de confianza en su posible contribución y aportación a la investigación, no obstante, esta circuns- 
tancia raras veces emerge con informantes de alta posición en donde suele darse con mayor frecuencia la construcción y presentación de un tipo ideal como ya hemos comentado.

Finalmente habría que hacer una reflexión acerca de la posición del propio investigador. Es importante evitar procesos de seducción y fetichización. El investigador tiene que resolver adecuadamente la interacción de subjetividades que al fin y al cabo se produce en este tipo de investigación.

En cualquier caso, el problema de la verdad es una cuestión un tanto difícil y suele resultar complicada en investigación cualitativa. Las narraciones suelen ser veraces cuando se utiliza la entrevista como estrategia metodológica puesto que la relación entre investigador e informante tiende a ser larga y profunda y el sujeto tiene necesidad de coherencia personal y social. Es cierto que la memoria suele conllevar algunos errores pero no lo es menos que los momentos culminantes de una vida son difíciles de olvidar y actúan como jalones a partir de los cuales se va reconstruyendo el pasado, y que debemos utilizar otros documentos para triangular la información y controlarla.

Las inquietudes y reflexiones metodológicas deben acompañar continuamente al investigador social que es consciente, por ejemplo, de que al principio no conoce el contexto necesario para comprender la perspectiva en la que está interesado. Además, surgen numerosas ambigüedades y contradicciones cuando se trata de recapturar el pasado en el contexto ya relativamente academizado que supone su mera presencia. Se trata de reflexiones con las que hay que seguir investigando y creando condiciones que hagan posible comprender el punto de vista de nuestros informantes y la lógica interna de la conducta que nos relatan porque, tal y como escribe Luisa Passerini, «como la entrevista es siempre el resultado de dos subjetividades que se encuentran en un cuerpo a cuerpo, nuestro lugar en la construcción de la memoria es esencial».

Concluyendo, en este tipo de investigación se combinan elementos documentales personales y sociales que son tanto reales como imaginarios y construidos de forma significativa. La memoria del informante debe ser entendida como una producción activa de significados e interpretaciones, de carácter estratégico y capaces de influir en el presente. Se trata de una «reflexión verbalizada de la verdad personal y de la realidad social $\rangle^{19}$, cuyo tratamiento debe ser minucioso, crítico y enriquecedor. El resultado dependerá de la capacidad y conocimiento por parte del investigador de las limitaciones y potencialidades que la oralidad y el material de vida aportan a la investigación biográfica.

19 Luisa Passerini en su obra de 1987, Fascism in popular memory. The cultural experience of the Turin working class. (CUP), acentuaba el carácter simbólico de la memoria: que es más una mediación simbólica que una reproducción de la realidad social. 


\section{BIBLIOGRAFÍA BÁSICA}

MARINAS, J. M.; SANTAMARIA, C. (1993), La historia oral: métodos y experiencias. Madrid, Debate.

De Miguel, J. (1996), Autobiografias. Cuadernos metodológicos, $\mathrm{n}^{\circ}$ 17. Madrid, CIS.

LÓPEZ-BARAJAS ZAYAS, E. coord. (1996), Las historias de vida y la investigación biográfica. Fundamentos y metodología, Madrid, UNED.

PujAdAs MuÑoz, J J. (1992), El método biográfico, el uso de las historias de vida en las ciencias sociales, Cuadernos Metodológicos, $\mathrm{n}^{\circ} 5$, Madrid, CIS.

SANMARTín, R. (2003), Observar, escuchar, comparar, escribir. La práctica de la investigación cualitativa, Barcelona, Ariel Antropología.

SANZ HernÁNDEZ, M. A. (2000), Ojos Negros, la memoria de un pueblo, Teruel, Instituto de Estudios Turolenses.

SChWARTZ, H.; JACOBS, J. (1984), Sociología cualitativa. Método para la reconstrucción de la realidad, México, Trillas. 